\title{
SiadEnv safety and communication features in real life scenarios
}

\author{
Trandabăț A. ${ }^{1, a}$, Pîslaru M. ${ }^{2, b}$,Trandabăț D. ${ }^{3, c}$ \\ ${ }^{1,2}$ Technical University “Gh. Asachi” of lasi, Faculty of Electrical Engineering, $23^{\text {rd }}$ Dimitrie \\ Mangeron, 700050, Iași, Romania \\ ${ }^{3}$ University "AI.I.Cuza" of Iasi, Faculty of Computer Science, Str. Gen. Berthelot 16, \\ 700483, Iași Romania \\ a ftranda@ee.tuiasi.ro, ${ }^{\mathrm{b}}$ mpislaru@ee.tuiasi.ro, ${ }^{\mathrm{c}}$ diana.trandabat@gmail.com
}

Keywords: intelligent building, wireless sensors network, forest fire detection system

\begin{abstract}
SiadEnv system was designed to keep track of the energy consumes in residential and industrial buildings. This will analyze and compute the energy consume real need in various scenarios. The main objective of SiadEnv is to reduce the energy losses by taking action and modifying the room settings. Thus, SiadEnv computes the difference between outdoor and indoor temperature and adjusts the heating or cooling management in order to maintain the comfort index and to reduce the energy consume. Moreover, it contains indoor safety modules that prevent or reduce the impact of unwanted events such as flood, fire, motion control (thief entry etc). Due to SiadEnv modular design based on wireless sensors networks, the fire monitoring safety module can be easy reconfigured in order to extend its applications. As further work, the SiadEnv safety module will be redesigned into a new application with important social economic and environmental impact, which will use monitor forest fire and predict its dynamic, in order to provide crucial data for forest salvation.
\end{abstract}

\section{Introduction}

The SiadEnv system is one intelligent home resource management system that aims to reduce the energy losses. SiadEnv is designed using several interconnected modules developed on wireless sensors network. The modules monitories different indoor utilities and using some predefined scenarios and real time information those can adjust the consumers parameters in order to cut off the unnecessary energy losses [1,5]The main modules are: thermal comfort module, illuminating module, communication module, electrical appliance assessment module, gaming module and not the last the indoor safety module. Due to the modules independent design, these can be easily reconfigured for different similar jobs in different scenarios.

The idea behind the SiadEnv system fits to the European context that creates the opportunity and the right policy for using the ICT technology in achievement of $2020 \mathrm{CO}_{2}$ reduction target [1]. The large market for one new system that aim to reduce the energy consume by combining communication technology, electronics and software was the engine behind SiadEnv that made this idea to came alive.

In this paper it will be presented the SiadEnv architecture, the Communication module and Security module.

\section{SiadEnv architecture}

The SiadEnv has two main components: hardware and software components. The hardware component is responsible for data acquisition, data transfer, manual and automatic control. The backbone of hardware configuration consists on a wireless network sensors configuration. The software module is responsible for data processing, protocol developing and all inputs and outputs control.

Hardware architecture As we mentioned above, the main part of the system is one wireless sensor network Fig. 1. In the experimental model it has been used two different wireless sensor network hardware. The main reason of this approach sits on the efficiency study that has been performing over the cost / ratability for the SiadEnv system. Experimenting the solution offered by 
National Instruments and by Libelium for Wireless sensor network the SiadEnv system includes both technologies in separate modules. Both configurations have similar hardware architecture with different settings based on one gateway and several programmable sensorial motes interconnected. Therefore the Libelium hardware based architecture will be detailed further on the SiadEnv Security Module description.

The NI architecture is compound from one NI 9792 Wireless Sensor Network (WSN) Programmable Gateway that is a LabVIEW Real-Time controller and a WSN gateway. The role of this gateway is to communicate with all WSN nodes and to run the SiadEnv algorithms in order to suggest to rooms occupants one action or to send an automatic control command to one of surveyed devices through the WSN nodes. The Gateway is wireless connected to the WSN nodes (WSNn). In our test system, we have used three different types of nodes: NI WSN-3226 that is a Programmable Voltage/RTD Combination Node with 4 channels, 20-Bit, NI WSN-3202 that is a programmable analog input Node with $4 \mathrm{Ch}, 16$-Bit, $\pm 10 \mathrm{~V}$ and NI WSN-3212 that is a programmable thermocouple input node with $4 \mathrm{Ch}, 24$-Bit. Each WSN node is wireless connected to each other, in that way forming an ad-hoc wireless sensor network.

All WSN are programmable nodes. In this way, they can be configured to maximize the battery live up to three years. This can be done mainly by configuring the communication protocol. There is no need to transmit over and over same value for a monitories parameter. Let take as example the temperature. The room temperature is a non-dynamic parameter. It changes the value in time relatively slowly. Due that, the node can transmit the temperature value to the gateway only when there is a change of temperature value or it can send the logged values with time stamp on a presented schedule. Moreover, the node can be configured to communicate with the nearest node in order to decrease the transmitting power. At the end, all WSN nodes have connectivity with the Gateway node direct or through other WSN node or nodes. To each WSN node are connected a series of sensors and controllable switches that enable it to monitories and to command several appliances such as lights, blinds, windows, air culling system, air ventilation system, heaters etc.

The programmable gateway is connected also to a building control unit that in SiadEnv architecture is compound from NI TPC-2515D that is a 15 inch LabVIEW Touch Panel Computer with Windows XP Embedded. From the building control unit, the building administrator can change some software settings in the SiadEnv system, can send some commands to WSN nodes, can monitories all the comfort parameters from all controlled rooms and can be obtained some statistical information from SiadEnv WSNn. This Building control unit is connected to a server that will allow the rooms user access over the internet to its room control settings. In this part is made also a comparative data storing in order to be able to extract at the end of season or when is needed some statistical information linked by the SiadEnv energy saving and by the building running costs

Software architecture The SiadEnv software is developed using several interconnected module. As it can be seen in the Fig. 2 the SiadEnv modules have to monitories the following parameters: thermal comfort, room occupancy, positioning, illumination, internal environment and electric power.

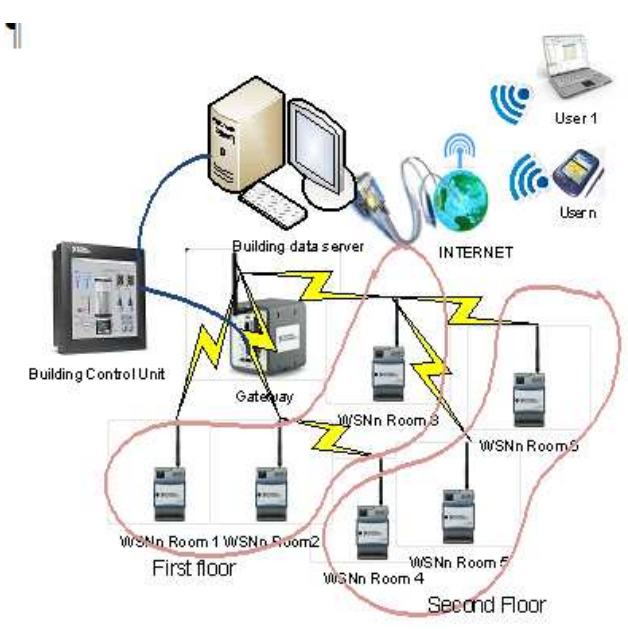

Fig. 1. SiadEnv Hardware architecture

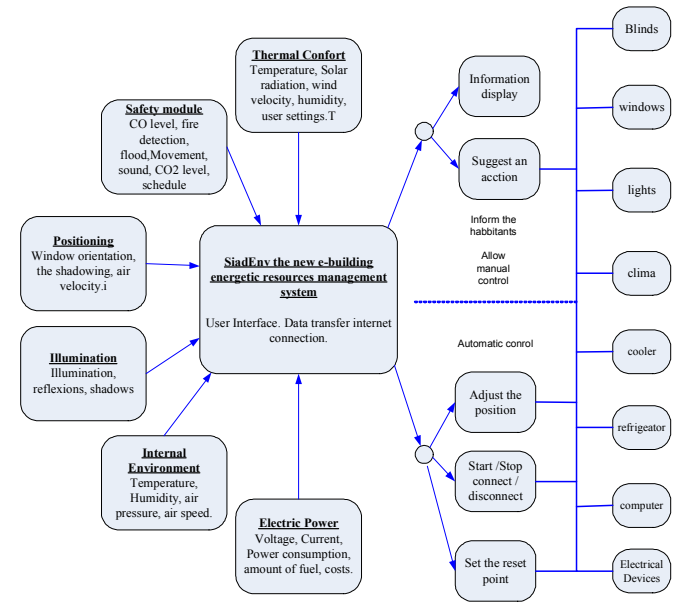

Fig. 2: The SiadEnv Software Architecture 
As output, SiadEnv has two different types of reactions informative one and automat action. The first output is just informative and is used only to inform the habitants allowing them to make their own decision and to manual control the room appliances. Here, the SiadEnv only will display some information, and will send some warning messages to the building administrator or to the room occupants. On the automatic control output, the system based on sensor inputs and room occupants' settings will control the room equipments, aiming to decrease consume. It will automatically change the indoor equipment settings, and in same time, it will be able to analyze and to record the human intervention over its settings, learning and applying these settings on next similar input conditions. The software has been developed under LabView graphical programming language and its modularity provides an easy maintenance, a high rehabilitee and a high confidence degree.

\section{SiadEnv COMMUNICATION MODULE}

The SiadEnv module for data transfer comprises the following modules: sensors to the server data transfer module, real time server data acquisition module, server historical data acquisition module and processing data module for server and local unit

Data transfer module taken from the sensors to a server consists of the following subprograms:

Sensors data acquisition module In the first experimental system we used one data acquisition board NI USB 6009 connected with current, voltage and temperatures sensors that aim to acquire data about electrical power consumption, indoor and outdoor temperatures values. This module aimed to provide data sets required to test the design and implementation of SiadEnv communication and processing modules for data transmission [5]. It has in its design one virtual power-meter that acquire the voltage and current information from one consumer and from those information are extracted the following data: $\cos ($ phi), power, active power and reactive power Also, it is computed and displayed the wave form for current and voltage. On this configuration, the system will need one electrical power sensing device for each electrical device. This approach is not willing to be implemented due to the implementation costs and due to the logistic that are involved by this solutions.

Another approach is to develop a smart meter [3]. It will have to monitories only one power line and will be able to identify the equipment in use connected to it by its electrical signature. Therefore, the smart meter will have to perform the signal disaggregation between individual devices or appliances and it will be able to measure different equipment or appliance consumes only using one set of sensors and only analog to digital converter for each room.

In order to perform this task it will need to include one software component that will analyze the global current and voltage waveforms, establish the load pattern of each powered appliances and compare this signature with those stored in a household appliances database. [4]

One method to perform this task is to use harmonic analysis to characterize the signature of electric devices. While linear loads draw currents of fundamental frequency, non-linear loads also draw harmonic currents. Analyzing their amplitudes and the values of harmonic real and reactive power, certain correspondences can be found between a type of device and its harmonic content.

Current waveform, instantaneous admittance waveform, instantaneous power waveform, eigenvalues were also used as features to characterize the electric load signature. Current waveform of a device has a different shape from that of another device according to its constituent components. Also because devices have different current consumptions, the instantaneous admittance and instantaneous power can offer information about the operating status of a device. The dynamics of variable loads can be observed by applying the eigenvalue analysis of the time series of the current waveform, rearranged into a matrix form.

One problem in identifying appliances is separating the power consumption of variable-speed drives. Because of their continuous variable power consumption, they can interfere in the process of identifying appliances with constant consumption. Therefore a new method was implemented, which uses correlations between fundamental and higher harmonic content to estimate the power consumption of a variable-speed drive.

In the final SiadEnv system configuration, the acquisition module based on data acquisition board it will be replaced by a virtual instrument that will retrieve data from a wireless sensory network formed in the first phase from four sensorial modules (sensorial nodes) and Gateway. It 
will be designed to retrieve process and transmit sensor data on the wireless network and it will control the functioning working times for some electrical appliances. For this reason I will not insist on the data acquisition module developed with NI USB-9006. This module output consist on three variables values for electrical power $\mathrm{kW}$ and values for indoor and outdoor temperature in degrees Celsius.

Message module for server data transfer - the solution used for SiadEnv data storage is the cloud storage solution offered by Pachube data server. The main allowance characteristics for Pachube data server are presented in table T1 Pachube data server accept data in its own XML format. Therefore in order to send our collected data to the Pachube server we need to use the following command syntax (fig.3):

\begin{tabular}{l|l}
\multicolumn{2}{c}{ T1 Pachube data server allowance } \\
\hline \multicolumn{2}{c}{ Pachube data server characteristics } \\
\hline Private and Public feeds & Unlimited \\
Number of data streams & Unlimited \\
Data point history storage & Unlimited \\
Data point bulk import & 500 per API request \\
Triggers & Unlimited \\
API usage limits & 100 requests per minute \\
\hline
\end{tabular}

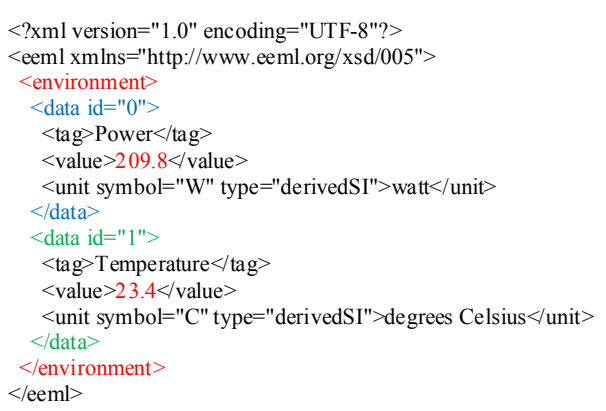

Fig 3 Pachube command syntax

The information collected from sensors that are included in the server message range between <environment> labels. Downloaded data contain information about the feeds label, value and measurement unit. To obtain the XML format recognized by the Pachube server are used three constant strings that are combined with measured values for power and temperature (input variables for this sub-VI), Fig. 4, which converted from integer format in text format result in a variable text message that will be used to write data on Pachube server in a separate sub-VI.
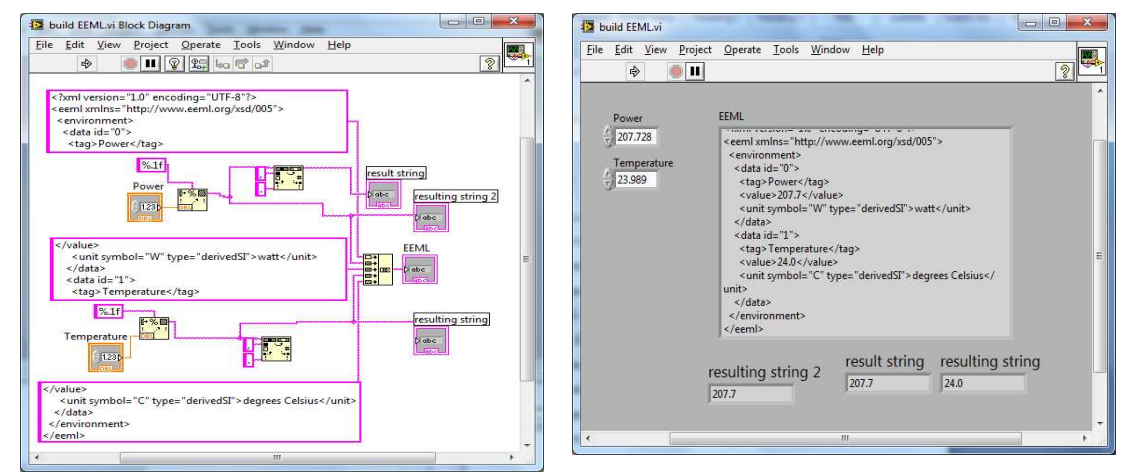

Fig. 4 XML data conversion module

The XML compiled data are used as input for the next sub-VI that main role is to put data on Pachube server. Therefore, in order to identify, call and modify your public feeds on Pachube server there are several API features available on Pachube data server. The information on Pachube server are structured in Public feeds that can be identified by a name (siadenv) and by a unique numeric code and each feed can contain an unlimited data streams that have one identification label (temperature or power etc) and unit. Each data stream contains numberless data points for each measured values. The maximum data points per minute can be up to 100 data points/minutes.

To access a user data channel (one feeds) can only find his name in the server search engine dialog line, or by using the keywords defined for the feeds. To write or to modify data in one feed data stream is requested to be login on Pachube server as feed administrator. To identify you as administrator for one feed, the Pachube server offer two identification methods: the classical method with the username and password associated with a data field or using a key automatically generated by Pachube server when a particular data field is created.

If there are needed some structural feed changes, or some manual data streams adjustments, the classical login method can be used, but in the case of automat data uploading is necessary to be used the second identification method based on automat generated feed access key. 
For this reason, the server message syntax must contain the following elements: the called server name, the number associated to the data field to be modified / completed, feed access key (42character string unique to each data field) and the XML parsed values

To concatenate all these data in LabView we choose the function "Format Into String" fig 5, which receives as input strings with the above information and has as output the server data write command PUT command in XML format (fig. 5):
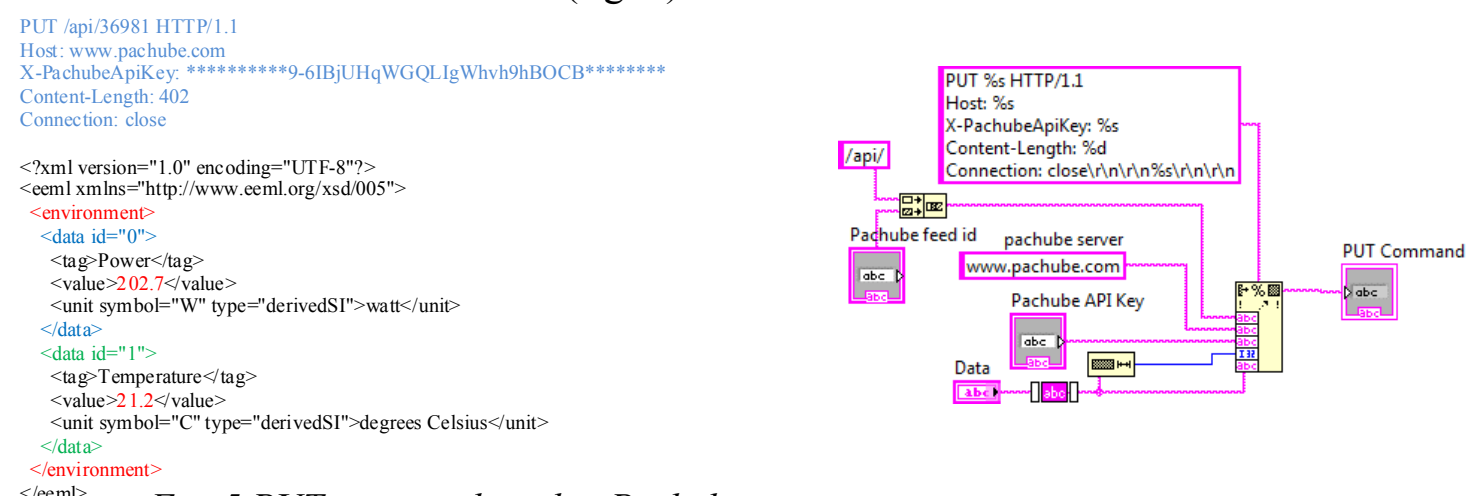

Fig. 5 PUT command used in Pachube server communication sequence

As shown the data field values and access key are entered manually, whereas the values for the "data" monitored values converted into XML are taken from previously described sub-VI.

The constant "pachube server" value is then used as input for the function that will establish TCP connections with the Pachube server using "TCP Open Connection" function Fig. 6

After the connection is established, if no error occur the PUT command is sent (Fig. 7) to the Pachube server using the "TCP Write" function which has as input string the XML parsed PUT command and references of previously established connection.

After sending the command to the server, it will take a reading of the data divided into packs of 1024 bytes until obtaining the first error. For this we used the "TCP Read" command which was included in a While structure to index its text output in order to extract the message from the server fig. 8. Loop is left at any error generated by the "TCP Read".
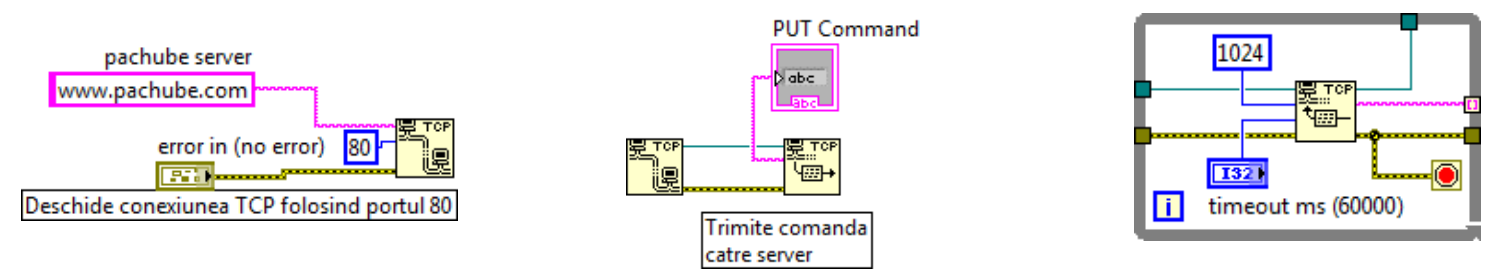

Fig 6 Pachube server connection syntax. Fig 7. Server request syntax Fig. 8 Server data reading syntax

One useful error is error code 66, which is generated at the end of data transfer. For this reason, this error should be treated differently from other errors and it should be treated as the end of data transmission. For this reason, the connection data containing information about the error and the error value is extracted if it is 66 will override the value of the output error data (Fig. 9).

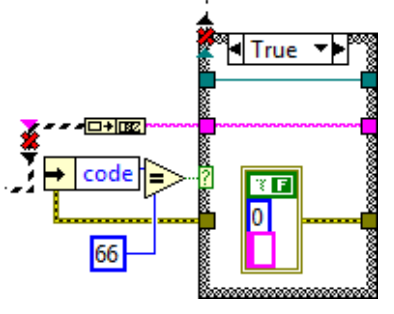

Fig. 9 Error cod 66 management

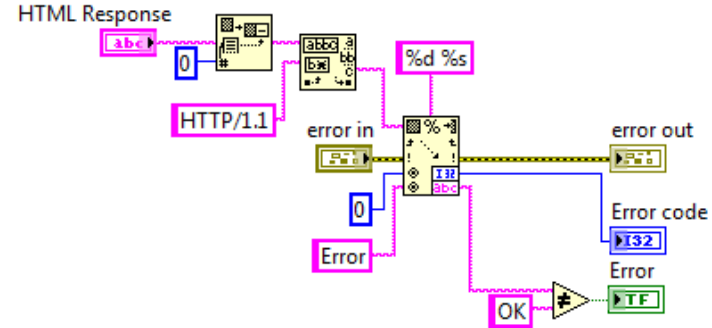

Fig 10 Server message parsing for error code extraction

In the last section the TCP IP connection is closed, and then the received message from the server is displayed. Here, in a separate sub-VI is parsed the text message received from the server (Fig. 10). The error value is extracted and it is displayed in one Boolean indicator which will have 
two states: red if an error occurs or green when data transfer was successful. This error display method is chosen in order to avoid that to do not be noticed by operator if it is displayed among other information.

The final message received from server is presented in fig 11

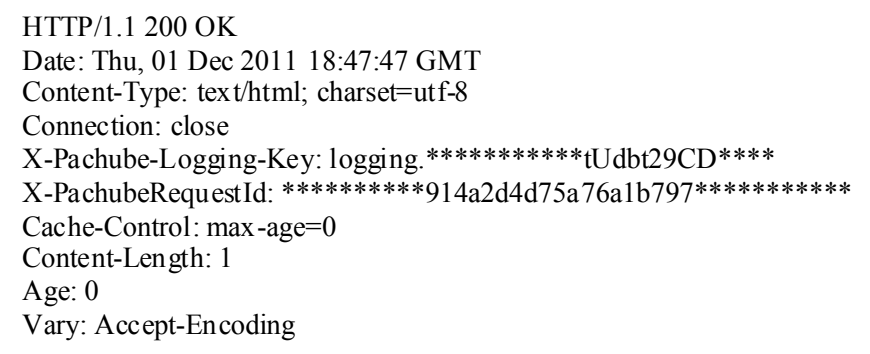

Fig. 11 Server acknoledge message

Historical data Pachube server querying module The structure of a query message for Pachube server history extraction is as fig. 12:

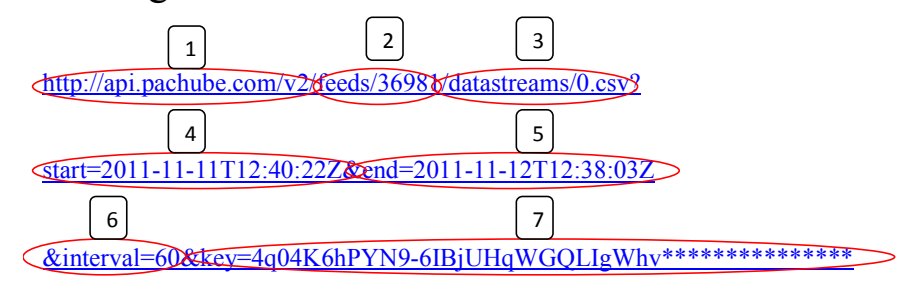

Fig. 12 Pachube server historical data querying message

Where 1. is server address, 2. is data field identification number 3. data set (a data field can have several data sets that are, identified by numbers $0,1,2$ etc.) and format the information required on the server: Csv Html or json, 4. is history interval retrieval starting date, 5. is last date for the history retrieval interval, 6 . is the interval between two consecutive records and 7 . It is the field data access key.

The user interface allows to the user to directly enter the passkey from keyboard, to introduce the identification number of data field and it assists the user in choosing the time to read history and delta $\mathrm{T}$ between two consecutive readings called. Limitations related to the delta $\mathrm{T}$ between two consecutive points extracted from the database server required by Pachube server are shown in Table T2.[7]

Table T2 Pachube server history query limitation

\begin{tabular}{l|l|c}
\hline \multicolumn{1}{c|}{ Value } & \multicolumn{1}{|c}{ Description } & Maximum range in one query \\
\hline $\mathbf{0}$ & Every snapshot stored & 6 hours \\
$\mathbf{3 0}$ & 30 second interval data & 12 hours \\
$\mathbf{6 0}$ & One snapshot every minute & 24 hours \\
$\mathbf{3 0 0}$ & One snapshot every 5 minutes & 5 days \\
$\mathbf{9 0 0}$ & One snapshot every 15 minutes & 14 days \\
$\mathbf{3 6 0 0}$ & One snapshot per hour & 31 days \\
$\mathbf{1 0 8 0 0}$ & One snapshot per three hours & 90 days \\
$\mathbf{2 1 6 0 0}$ & One snapshot per six hours & 180 days \\
$\mathbf{4 3 2 0 0}$ & One snapshot per twelve hours & 1 year \\
$\mathbf{8 6 4 0 0}$ & One snapshot per day & 1 year \\
\hline
\end{tabular}

To implement this restriction table were used for constructing a series of "Case" structures fig. 13 filters in series in order to use the reverse principle of decreasing grain size. The structure check in which the query interval fit in (maximum 6:00, 12:00, etc.) It is checked delta $\mathrm{T}$ between two consecutive values, and if it is less than the amount allowed by the server is automatically decreased to the minimum value supported by server.

The user assistant component for history scale extraction choice from the database verifies that requests made are valid requests (TStop - TStart $<0$ ). If the request is negative, the system will consider the date of completion of the query, and sets the start dates of the period the first interval 
date decreasing from it 6 hours in order to establish the final moment for the Pachube server history query. However, the system warns the user about the automatically made changes, still leaving him to choose other values for the start or end dates. Implementation of the Sub-VI was performed using the structure "Case" and time and data type specific functions Fig. 14.

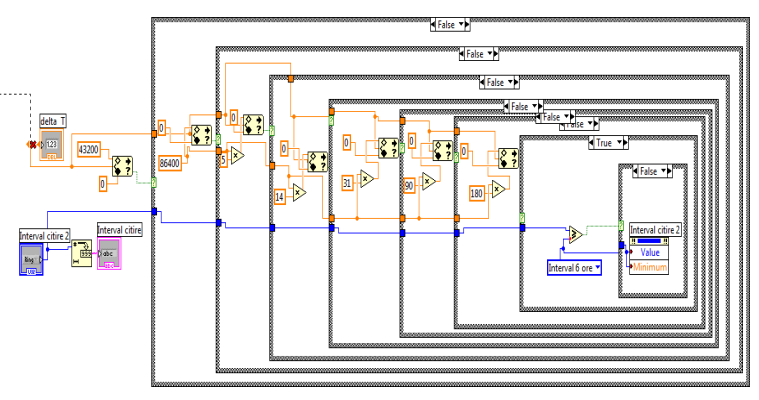

Fig. 13 Time interval selection assistant module

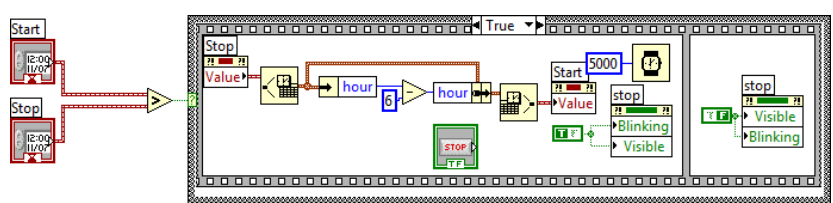

Fig. 14 History data interval selection syntax

\section{WEB interface}

The SiadEnv data from Pachube server can be visualized on the project webpage as graphs for temperature and power. On the SiadEnv web page (fig. 15) http://www.siadenv.pht.ro/siad.html the user can choose to see the information displayed for last 24 hours, for last hour and for the last month as graphical representation.

As further approach, on the same webpage will be included one portal that will allow to different users to consult their own consumes concomitant with other consumers that use the SiadEnv resources. The aim of this approach is to reduce furthermore the energy consume by using the gaming theory [6]. According to this approach, the humans are in continuous contest.

There are some psychological studies that have demonstrated that if there is a reword or there is a competition then there is a motivation for humans to do their best. In this context, will be developed one web platform that will allow gaming scenarios. By seeing in some statistics the position of your energy consume compared to the neighbors consume, it can be developed a gaming strategy based on points, lives, achievements, etc... that will motivate further on the SiadEnv users to reduce the energy consume not only because of energy costs but because of the willing to overcame and win the other "contest" participants.

\section{SiadEnv Security Module}

According to recent researches [8] one of the major domestic accidental death reasons is the CO intoxication poisoning. Another important percentage of accidental deaths occurred in residential buildings is the fire [9]. Regarding the in home accidents from the damages perspective, the most common accident is represented by the floods, followed by fire.

Therefore, the SiadEnv security module is designed to keep under constant supervision floods, fire, $\mathrm{CO}$ level unauthorized in home access in order to alert and to minimize the consequences.

The SiadEnv security module is based on the Libelium Wireless sensor network (WSN). For each room are used two motes equipped with different sensors: $\mathrm{CO}$ sensor, $\mathrm{CO}_{2}$ sensor, $\mathrm{CH}_{4}$ sensor, Humidity sensor, pressure sensor, water presence sensor, temperature sensor, volatile organic compounds sensor, presence sensor, ultrasonic sensor and Dust - PM-10 sensor. The WSN motes are interconnected and communicate together through similar motes or directly with the SiadEnv gateway. The software deployed on the motes is designed using two main demands: to increase the motes batteries life and to increase the indoor safety.

The part of SiadEnv safety module that surveys the fire has a common part with the dangerous gases monitoring sub-module. Therefore the $\mathrm{CO}, \mathrm{CO}_{2} \mathrm{CH}_{4}$, temperature, volatile compounds sensors and Dust - PM-10 sensor are used with double purpose. To reduce the online time of the SiadEnv safety module motes, there are implemented 4 algorithms on each mote, which are responsible for acquiring data values, comparing with the previous values and running the algorithm for threat estimation. If one or more acquired parameters differ in a predefined range from previous acquired value then the threat estimation algorithm is activated. It will compute the risk of fire 
occurrence and the air quality. If there are some important changes then the alert message is send to SiadEnv main unit. Here, depending on the type of threat, the SiadEnv will activate the appropriate alarm. The SiadEnv can be configured to send alarm messages with a high confidence rate to different authorities and to the building manager or to the owner. The message contain information about the type of alarm, the starting time of alarm, the building address and the estimated number of persons at the address on the alarm triggering moment. If on site there is an automatic fire prevention system then the SiadEnv can activate it automatically. If the threat is represented by a gas leak, or by CO, concomitant with the alarm the SiadEnv security system cut the gas alimentation and activate the ventilation module that will help on the rapid air decontamination.

The indoor water flood monitoring module has in its composition water presence sensors arranged near the sinks or near the other water sources. This module combines its information with the acquired row data from the SiadEnv thermal comfort module (indoor temperature, humidity). If the water is sensed by security WSN motes sensors that are deployed in normal dried spots then the main water admission valve is activated in order to close the main water vane.

The safety perimeter guarding module is responsible for people tracking inside the building. Its output is use in several different SiadEnv modules that track the energy consumption and compute the best economical scenarios for room illuminating or for thermal comfort module. Its main functionality is to act as an unauthorized room entering alarm. This functioning mode can be triggered manually by house user or automatic by using verbal commands integrated in speech commands recognition module. If during the watching mode one alarm is triggered by an unauthorized room access, then are several scenarios that can occur, depending on system settings. The first step on alarm mode is to send one SMS alarm message to the owner sending the alarm code time and number of unauthorized persons that entered in the monitories area. If the owner decides that the alarm is real, then with an SMS command to SiadEnv sets it on the second stage of perimeter unauthorized access alarm. On this alarm stage, the SiadEnv system sends one distress alarm messages to authorities. This message can be sent using SMS or SiadEnv communication module through internet. If the pressure sensors installed on potential access points (doors, windows) or the hall sensors who monitories the closure of the access points sense that one room entering point was force, then the system is automatic set to the second alarm stage. The design of the safety perimeter guarding module is based on Libelium WSN motes. It contains the events sensor board, specially equipped for perimeter monitoring with pressure sensors, ultrasonic indoor sensor, bend sensors, hall sensors, presence sensor and optic barrier. Also it uses information about $\mathrm{CO}_{2}$ level from gases monitoring motes. The perimeter monitoring algorithm is triggered after this function is activated on SiadEnv by value changes occurred in one or more surveyed parameters.

SiadEnv security module hardware configuration is developed using Libelium WSN solutions. Each SiadEnv security module mote has a commune configuration and a specific one depending on its functionalities. The backbone of SiadEnv security mote is compound from Wapmote fig. 15, power supply and the communication module XBee-ZB-Pro fig. 16 which can have different sensor boards with different sensors array installed.

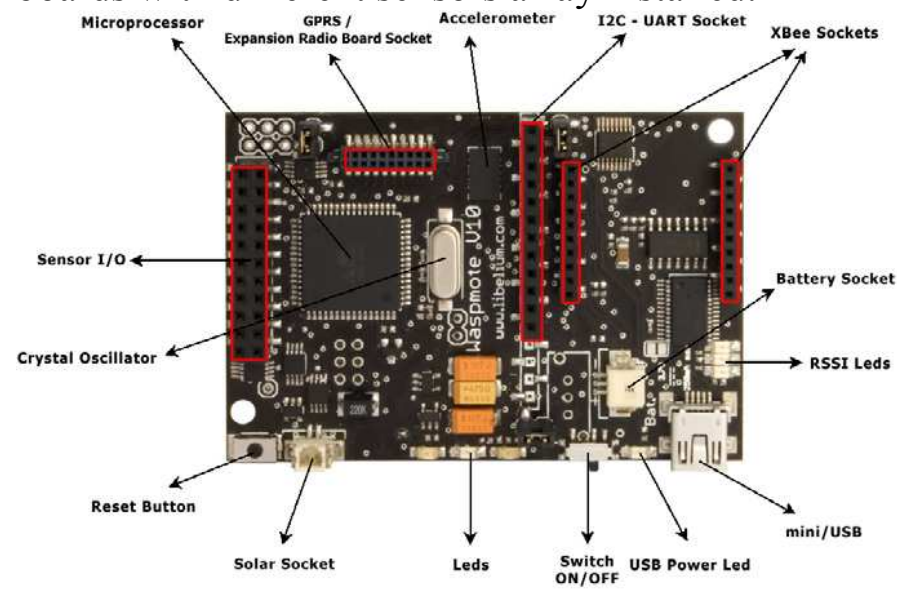

Fig. 15 Waspmote board

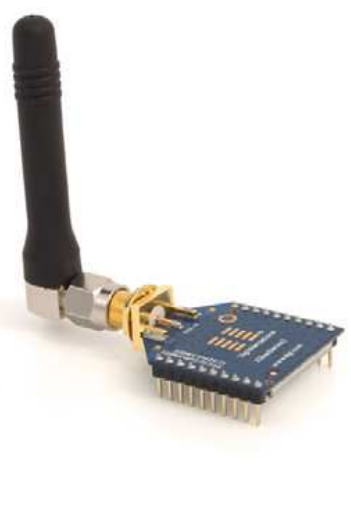

Fig. 16 XBee-ZB-Pro 
The Waspmote characteristics are shown in table 3 and the XBee-ZB-Pro characteristics are presented in table 4

Table 3 Waspmote caracteristics

\begin{tabular}{ll}
\hline Microcontroller & ATmega1281 \\
\hline Frequency & $8 \mathrm{MHz}$ \\
SRAM & $8 \mathrm{~KB}$ \\
EEPROM & $4 \mathrm{~KB}$ \\
FLASH & $128 \mathrm{~KB}$ \\
SD Card & $2 \mathrm{~GB}$ \\
Weight & $20 \mathrm{gr}$ \\
Dimensions & $73.5 \times 51 \times 13 \mathrm{~mm}$ \\
Clock & RTC (32KHz) \\
Inputs/Outputs & $7 \mathrm{Analog}(\mathrm{I}), 8 \mathrm{Digital}(\mathrm{I} / \mathrm{O}), 1$ \\
& PWM, 2 UART, 1 I2C, 1USB \\
\hline
\end{tabular}

Table 4 XBee-ZB-Pro characteristics

\begin{tabular}{ll}
\hline Antennas & $\mathbf{2 . 4 G H z} \mathbf{2 d B i} / \mathbf{5 d B i}$ \\
\hline Connector & RPSMA \\
Encryption & AES 128b \\
Control Signal & RSSI \\
Standards & ZigBee-Pro v2007 Compliant \\
Topologies & star, tree, mesh \\
\hline
\end{tabular}

The sensors boards used in the security module configuration are: gas sensor board events sensor board smart metering sensor board and smart city sensor board.fig. 17

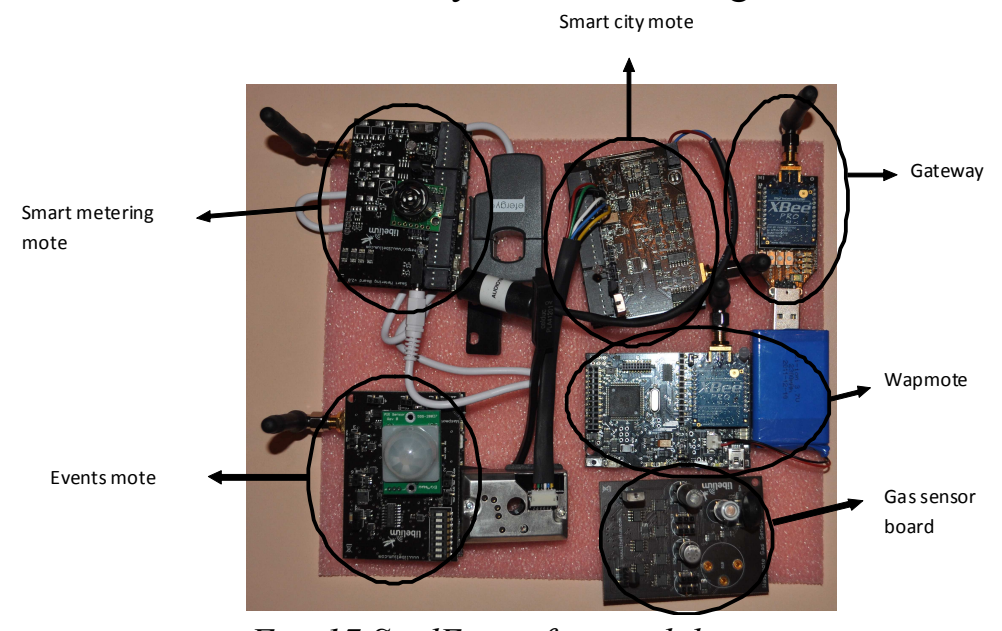

Fig. 17 SiadEnv safety module

\section{Further work}

The next step on the SiadEnv security module upgrading process will be to integrate the events recorded on SiadEnv database in to one self tuning program. This program will be realized using neuronal networks algorithms that will learn from the alarm database and from the values database which situation are real threats and what values can set a false alarm. This element will increase the alarms confidence values and will reduce the false alarms. Moreover, using as input the microphone from WSN motes will be developed speech recognition software that will recognize some keywords which will allow to system to reevaluate the situation. For example the affirmation "I feel smog" can reactivate the fire detection module that will reevaluate the fire occurrence.

Due to the climatic changes, one of the major problems that affected the world by economical, social and material point of view was the forest fires. The security module of SiadEnv provides the main elements for fire detection, prevention and with minor adjustments can provide information about its dynamics. This is the starting point for a new independent application on field of forest fire detection, prevention and assessments that will be developed as further work of the project 4DPostdoc. The idea of forest watching system has its start based on the huge potential market of this new system. This new system is derivate from SiadEnv security module. It aim to survey the forest and to provide critical information about fire occurrence, fire dynamics, fire localization and about the tree harvesting region from forest in order to detect the illegal tree cuts from the forests. Moreover, using the microphone sensor can be implemented one forest noise analyzer algorithm through can be discovered the shot gun and the chainsaw noises. Using the localization of those noises, through WSN can be reduced two forest plaques: the illegal tree cuts and the illegal wild animal hunting. 


\section{Conclusions}

The SiadEnv system is the backbone for intelligent house. It aims not only to increase the users comfort and to decrease the energy consume but to ensure the house safety. Due that, in this paper it is presented the SiadEnv architecture, the communication and the security module. It has been identified two possible upgrades that can be applied to the SiadEnv security module, upgrades that will increase the security alarm confidence.

Due to SiadEnv modular design based on wireless sensors networks, the fire monitoring safety module can be easy reconfigured in order to extend its applications. As further work, the SiadEnv safety module will be redesigned into a new application with important social economic and environmental impact, which will use monitor forest fire and predict its dynamic, in order to provide crucial data for forest salvation.

The SiadEnv System is a new approach in the battle for resources savings. It combine the ITC domain capabilities in order to reduce the unnecessary consumes.

\section{Acknowledgment:}

This paper was supported by the project "Development and support of multidisciplinary postdoctoral programmes in major technical areas of national strategy of Research - Development Innovation" 4D-POSTDOC, contract no. POSDRU/89/1.5/S/52603, PERFORM-ERA ID - 57649 POSDRU/89/1.5/S/57649 projects co-funded by the European Social Fund through Sectoral Operational Programme Human Resources Development 2007-2013 and by project CNCSIS PD contract 55/2011

\section{References}

[1] Trandabat A, Pislaru M, Brinzila M. SiadEnv - Environmental friendly e-building energy management system ENVIRONMENTAL ENGINEERING AND MANAGEMENT JOURNAL Volume: 11 Issue: 3 Pages: 687-694, ISSN 1582-9596

[2] Pislaru M, Trandabat A, Avasilcai S Neuro-fuzzy model for environmental impact assessment ENVIRONMENTAL ENGINEERING AND MANAGEMENT JOURNAL Volume: 10 Issue: 3 Pages: 381-386 ISSN 1582-9596

[3] Baranski M, Voss J Detecting Patterns of Appliances from Total Load Data Using a Dynamic Programming Approach, Proceedings of the Fourth IEEE International Conference on Data Mining, 227-330, 2004

[4] Liang J., Ng S.K.K., Kendall G., Cheng J.W.M., Load Signature Study-Part I: Basic Concept, Structure, and Methodology, IEEE Transactions on Power Delivery, Vol. 25, No. 2, pp. 551560,2010

[5] A. Trandabat, M. Pislaru New energy management tool for buildings active energy consumes reduction Proceedings of the 7th International Conference on Management of Technological Changes ISBN (Book1): 978-960-99486-2-3,2011, pp.617-620.

[6] M. Pislaru, A. Trandabat Decision suport tool based on neuro-fuzzy environmental approach Proceedings of 2nd International Conference on Environmental Engineering and Applications, 2011, ISSN 2010-4618; pp 68-73

[7] https://cosm.com/docs/ active on January 2013

[8] Stefanidou ME Maravelias CP Dona AA Pistos CM Spiliopoulou CA Athanaselis SA Carbon Monoxide-Related Deaths in Greece A 23-Year Survey, AMERICAN JOURNAL OF FORENSIC MEDICINE AND PATHOLOGY Volume: 33 Issue: 2 Pages: 128-131 DOI: 10.1097/PAF.0b013e318252eca9 ISSN: 0195-7910

[9] Holborn PG Nolan PF Golt J An analysis of fatal unintentional dwelling fires investigated by London Fire Brigade between 1996 and 2000 FIRE SAFETY JOURNAL Vol. 38 Issue 1 Pages 1-42, ISSN 0379-7112 